Www.jmscr.igmpublication.org

Impact Factor (SJIF): 6.379

Index Copernicus Value: 71.58

ISSN (e)-2347-176x ISSN (p) 2455-0450

crossrefDOI: https://dx.doi.org/10.18535/jmscr/v6i7.154

Journal Of Medical Science And Clinical Research

\title{
Neonatal ICU working diagnosis of Neonatal Necrotising Enterocolitis: The Paediatric Surgical perspective
}

\author{
Authors \\ Dr Vivek Parameswara Sarma, Dr Sunil S Menon \\ Department of Paediatric Surgery, SAT Hospital, Govt Medical College, Thiruvananthapuram
}

\section{Introduction}

A neonate with features of intestinal obstruction continue to baffle experienced neonatologists and even paediatric surgeons. Hence, when a neonate with such features is admitted to an NICU, it has been observed that the resident physician makes a working diagnosis of NNEC in many cases. We observed that the prelimniary diagnosis may bias subsequent judgement and management in few cases where the diagnosis is unwarranted and actual pathology are different.

\section{Aims}

The aim of the study was to analyse the actual pathologies in cases of neonatal intestinal obstruction, which were initially treated with a working diagnosis of NNEC in the Neonatal ICU, and later proven to be other causes during further evaluation. [Refer Figure 1]. An attempt was also made to define factors which would help differentiate the other possible surgical / medical causes.

\section{Methods}

A Retrospective analysis of neonates referred for surgical opinion from the neonatal ICU with a preliminary diagnosis of NNEC and detected to have another major surgical / medical problem on evaluation / surgery during the period of Jan 2011 to Jan 2016.

20 Cases were selected for analysis based on availability of all necessary data. All cases had an initial working diagnosis of NNEC before being referred for surgical opinion. [Refer Table 1].

Table 1 : Clinical Presentation

\begin{tabular}{|l|c|}
\hline Common presnting features & $\mathbf{N = 2 0}$ \\
\hline Abdominal distension & 17 \\
\hline Non passage/ Delay in passage of meconium & 15 \\
\hline Bilious vomiting/ bilious nasogastric tube aspirate & 14 \\
\hline Blood in stools & 4 \\
\hline Antenatal polyhydramnios & 2 \\
\hline Maternal diabetes mellitus & 5 \\
\hline Maternal hypothyroidism & 4 \\
\hline
\end{tabular}

The clinical records, investigation reports, surgical notes and post operative findings, in cases which were operated, were reviewed.

[Refer Table 2].

Table 2 : Radiological findings including plain radiograph and contrast enema

\begin{tabular}{|l|c|}
\hline $\begin{array}{l}\text { Radiological findings including } \\
\text { contrast enema in 13 cases }\end{array}$ & $\mathbf{N = 2 0}$ \\
\hline Proximal gut obstruction & 2 \\
\hline Distal small bowel obstruction & 7 \\
\hline Large bowel obstruction & 6 \\
\hline Non specific & 5 \\
\hline
\end{tabular}




\section{Results}

On detailed evaluation in all cases and surgery in select cases, the final diagnosis was established to be other significant medical/surgical in these cases including [Refer Table 3];

\begin{tabular}{|ll|}
\hline & Hirschsprung's disease \\
- & Hypothyroidism \\
- & Meconium atresia \\
& perforation \\
- & NSLCS \\
- & Malrotation with Midgut Volvulus \\
- & Meconium ileus \\
\hline
\end{tabular}

Table 3 : Final diagnosis in cases where the initial working diagnosis was NNEC

\begin{tabular}{|l|c|}
\hline Final diagnosis & N=20 \\
\hline Hirschsprung's disease & 4 \\
\hline Hypothyroidism & 4 \\
\hline leal atresia & 3 \\
\hline $\begin{array}{l}\text { Meconium Peritonitis / Idiopathic } \\
\text { Bowel perforation }\end{array}$ & 3 \\
\hline Neonatal Small Left Colon Syndrome & 2 \\
\hline Malrotation with Midgut Volvulus & 2 \\
\hline Meconium ileus & 1 \\
\hline Meconium plug syndrome & 1 \\
\hline
\end{tabular}

The factors predictive of a pathology other than NNEC were

1. Absent risk factors of NNEC like prematurity, low birth weight, perinatal complications or formula feeding ${ }^{(8)}$

2. Atypical History, not fitting that of typical $\mathrm{NNEC}^{(7)}$

3. Absence of physical findings typical of NNEC like abdominal wall erythema or blood in stools $^{(7)}$

4. Improperly done/read initial radiograph ${ }^{(9)}$

5. Absent evidence of NNEC on radiograph (pneumatosis intestinalis/portal venous gas/ pneumoperitoneum) $)^{(10)}$

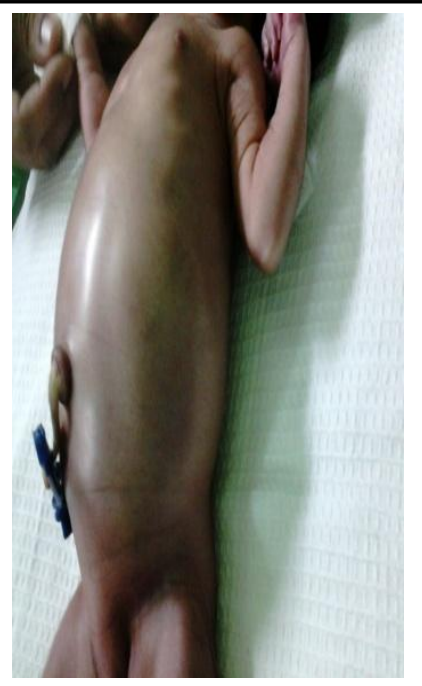

Figure 1 : Meconium peritonitis

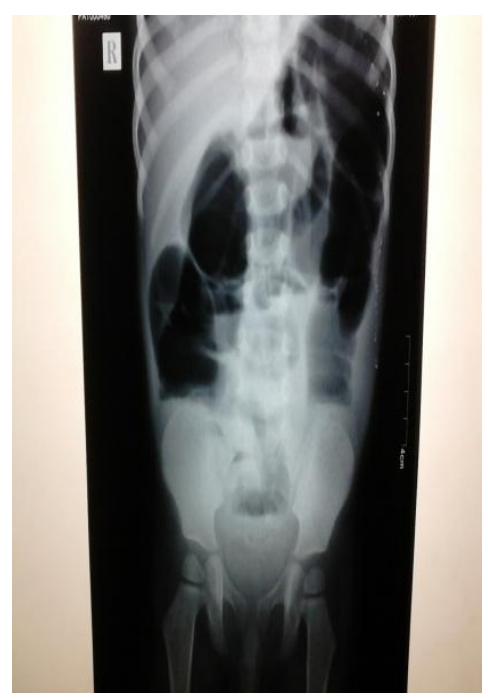

Figure 2 : Meconium Plug Syndrome

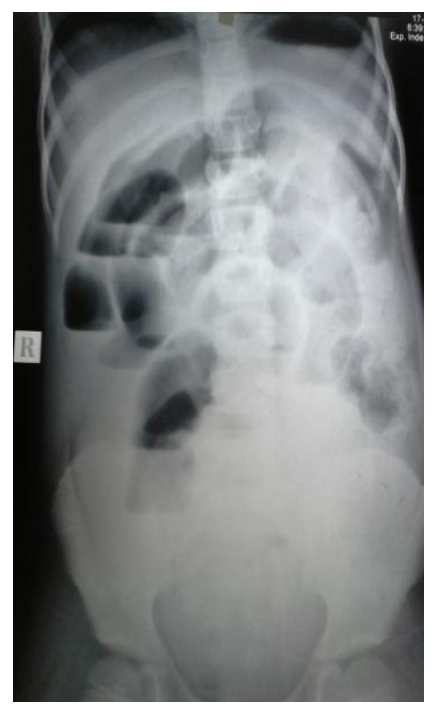

Figure 3 : Hirschsprung's disease 


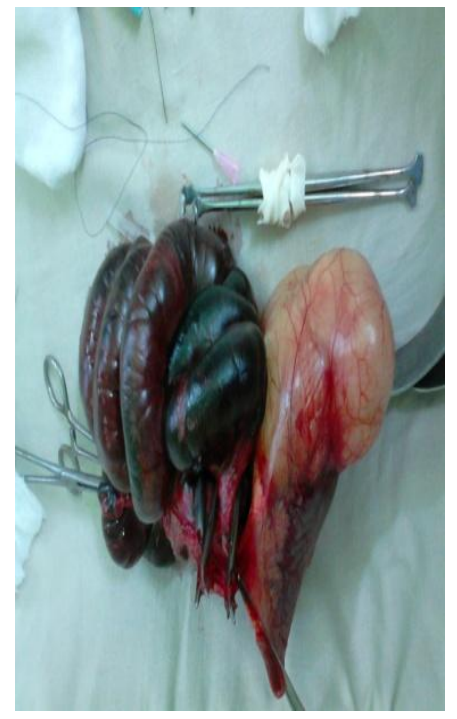

Figure 4 : Malrotation with Midgut Volvulus

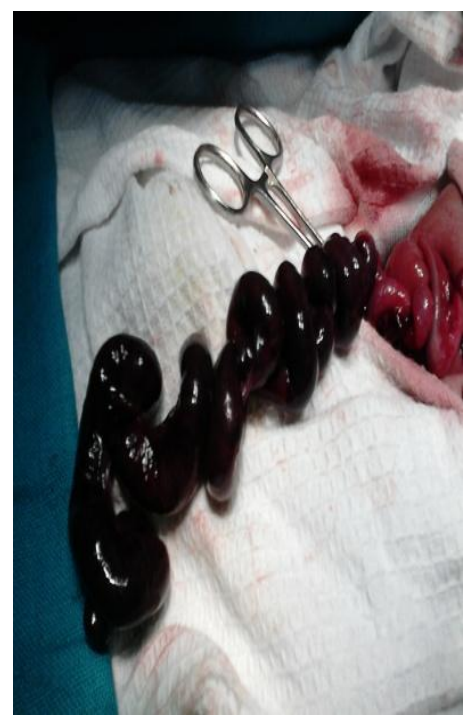

Figure 5 : Type IIIb Apple peel Atresia

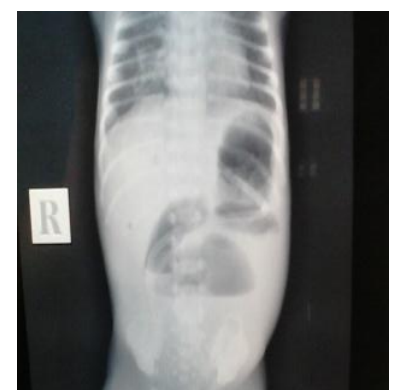

Figure 6 : Ileal Atresia

\section{Conclusions}

A high index of suspicion is necessary while attending a patient treated for suspected NNEC. Exclusion of usual risk factors for NNEC, a proper interpretation of radiograph and targeted evaluation will clinch the diagnosis.

\section{References}

1. Neonatal Necrotizing Enterocolitis: Diagnosis management and Pathogenesis Yost, Christian Con MD, Journal of Infusion Nursing March - April 2005, Vol 28- Issue 2 P 130-134

2. The Micro Biome and Biomarkers of Necrotizing Enterocolitis: are we any closer to Prediction? RusconiB, Good M, Warner BB. J Pediatric. 2017 Oct . 189:40-7.e2.

3. Neonatal necrotizing enterocolitis, an epidemiological study NarangA, Rao R, Bhakoo ON. Indian Pediatrics 1993; $30: 1207-1214$

4. Changing trends in Neonatal necrotizing enterocolitis. Experience of 302 cases in 2 decades Grosfeld, JL, Gehu H, Schlatter M, West KW, Rescorla FJ Ann Surg 1991; 214: 300-306 discussion 306-307

5. Epidemologic study of Neonatal necrotizing enterocolitis among low birth weight infants. Absence of identifiable risk factors. Kliegman RM, Hack M , Jones P , Fanaroff AA. J Pediatrics 1982; 100:; 440-444

6. Necrotizing enterocolitis (NEC) in Newborn Hormann M, Pumblerger W, Puig S, Kreuzer S, Metz VM Radiologe 2000, Jan ; 40(1); 58-62.

7. Predictors of Gangreneous necrotizing enterocolitis and extent of disease. Early laparotomy versus peritoneal drainage Roy A Tayeb M, Khogeer SS, Al Salem AHSaudi Med J. 2005 Mar; 26(3): 447-52

8. Necrotizing enterocolitis and volvulus in prematre neonate Boulton JE, Ein $\mathrm{SH}$, Reily BJ, Smith BT, Pape KE.J Pediatric Surg. 1989 Sept; 24(9) : 901-5.

9. Stehr M, Lehner M, Schuster T, Heinrich M, Dietz HG. Tubularized incised plate (TIP) urethroplasty (Snodgrass) in primary hypospadias repair. Eur J Pediatr Surg. 2005;15:420-4 Surgical necrotizing enterocolitis Robinson JR, Rellinger EJ, 
Hatch LD, et al.Seminars Perinatol 2017;

$41: 70$

10. Neonatal necrotizing enterocolitis Theraputic decisions based up on clinical staging Bell MJ, Tern Berg JL, Feigin RD, et al.Ann Surg 1978; 187:1. 